\title{
Assessing wind profile effects on the global atmospheric torque
}

Article

Accepted Version

Miranda, P. M. A., Martins, J. P. A. and Teixeira, M. A. C. (2009) Assessing wind profile effects on the global atmospheric torque. Quarterly Journal of the Royal Meteorological Society, 135 (640). pp. 807-814. ISSN 1477870X doi: https://doi.org/10.1002/qj.393 Available at https://centaur.reading.ac.uk/29243/

It is advisable to refer to the publisher's version if you intend to cite from the work. See Guidance on citing.

Published version at: http://dx.doi.org/10.1002/qj.393

To link to this article DOI: http://dx.doi.org/10.1002/qj.393

Publisher: Royal Meteorological Society

All outputs in CentAUR are protected by Intellectual Property Rights law, including copyright law. Copyright and IPR is retained by the creators or other copyright holders. Terms and conditions for use of this material are defined in the End User Agreement.

\section{www.reading.ac.uk/centaur}

\section{CentAUR}

Central Archive at the University of Reading 
Reading's research outputs online 
Assessing wind profile effects on the global atmospheric torque

P. M. A. Miranda, J. P. A. Martins, and M. A. C. Teixeira

University of Lisbon, CGUL, IDL, Lisbon, Portugal

pmmiranda@fc.ul.pt

submitted to the Quarterly Journal of the Royal Meteorological Society

10 October 2008

Revised, 13 January 2009 


\section{Abstract}

The impact of a new approach to the evaluation of surface gravity wave drag (GWD) is assessed. This approach uses linear theory, but incorporates the effects of wind profile shear and curvature, by means of a second order WKB approximation. While the theory predicts the possibility of either drag enhancement or reduction, depending on the wind profile, results obtained with the ERA-40 data clearly indicate the predominance of local drag enhancement. However, the global impact of shear on the atmospheric axial GWD torque comes mostly from regions with predominantly easterly flow, contributing to a slight reduction of the bias found in different studies of the global angular momentum budget. The relative correction due to shear on linear GWD is found not to depend too strongly on the levels chosen for the computation of the low level wind derivatives.

\section{Introduction}

After the studies of Palmer et al. (1986) and McFarlane et al. (1987), gravity wave drag (GWD) is parameterized in all global models. The need for the inclusion of the effect of subgrid scale topography in the drag was suggested much earlier on theoretical grounds (Sawyer, 1959). But its development was motivated by the practical need to reduce a strong westerly bias in the northern hemisphere winter circulation, associated with a too weak northward heat transport, found in Numerical Weather Prediction models at that time. The attribution of the bias in the mean zonal wind to orographic effects was strongly supported by one of the first systematic studies of medium range forecast statistics, made by Wallace et al. (1983), using ensembles of 10-day forecasts made by the ECMWF model. That study, 
however, proposed to use an enhanced version of the mean orography (envelope orography) to amplify its effects on the flow.

The design of GWD parameterization has evolved significantly since its introduction. Initially, models used results from 2D linear theory to compute the surface drag, and the concept of gravity wave saturation (Lindzen, 1981) to evaluate the deposition of the wave momentum flux into the mean flow. Later models (e.g., Lott and Miller, 1997; Gregory et al.,1998; Kim and Doyle, 2005) tended to incorporate 3D and non-linear effects, namely high-drag regimes and low level blocking, and to improve the momentum deposition algorithms by including boundary layer and even directional filtering effects (Shutts and Gadian, 1999).

In spite of those improvements, generally well supported by mesoscale numerical simulations of flow past idealized (Stein, 1992; Miranda and James, 1992) or real topography (Doyle and Jiang, 2006), and of the fact that GWD parameterization did reduce the westerly bias, the impact of GWD on the atmospheric angular momentum budget is still not well understood. Indeed, Huang et al. (1999), based on NCEP/NCAR reanalysis, found a large bias in that budget, and, more importantly, concluded that the inclusion of the GWD term seemed responsible for a large fraction of the bias.

The relevance of global angular momentum budget for long term climate simulations cannot be overemphasized. At the global scale, angular momentum is one of the few variables that should be (almost) conserved, and its seasonal variations can be checked by independent astronomical measurements of the Earth's rotation rate (e.g., Egger et al., 2007). On the other hand, because most of GWD comes from the large mountain ranges, which are located far away from each other, the understanding of the regional patterns of GWD and corresponding interactions with the regional atmospheric circulation (Egger et al., 2007; Smith et al., 2007) 
is as important as the global balance. The relevance of GWD in the stratospheric circulation is also well established (e.g., Kim, 2007).

The large bias found in atmospheric reanalysis clearly indicates that we are missing some important effects in the angular momentum budget, in spite of the increasing complexity in the parametrization schemes. In the present paper we address one of the missing effects in current schemes: vertical wind shear. A number of recent studies have clarified the impact of shear on GWD, by extending linear theory to include shear effects. Teixeira et al. (2004) developed a second order WKB approach, showing that wind profile shear and curvature can have a significant impact on the drag, a result that was well reproduced by a non-hydrostatic mesoscale model. The theory was later extended to the case of flow past an elliptical mountain (Teixeira and Miranda, 2006), in a form that is readily usable for parametrization.

Other studies have considered the impact of shear on GWD, namely Kim and Arakawa (1995) and Shutts and Gadian (1999). However, those studies did not consider its impact on the surface drag, but on the vertical distribution of flow deceleration, another important aspect of the GWD process that is beyond the scope of the present study.

In the present paper we apply the theory developed by Teixeira and Miranda (2006) to compute global fields of surface GWD, using ERA-40 reanalysis data (Uppala et al., 2005). Section 2 describes the method. Section 3 presents the main results, followed by a short discussion in Section 4.

\section{Model and methods}

Parametrization schemes require an estimation of surface GWD. This is generally computed from linear theory, albeit with corrections to take into account non-linear effects, such as flow blocking and wave breaking. In the case of the parametrization scheme used by ECMWF in 
ERA-40 (Lott and Miller, 1997), the linear component is computed by application of Phillips (1984) linear model of inviscid, hydrostatic flow past an isolated, bell-shaped, elliptical mountain. Teixeira and Miranda (2006) extended Phillips theory to include the effect of wind shear, through a second order WKB approach. While the theory is formally valid for weakly sheared flows, numerical results indicate that it is applicable for flows with significant shear, e.g. for Richardson numbers as low as 0.5. Following Teixeira and Miranda (2006), the effect of wind shear on GWD can be computed as a multiplicative algebraic correction to the drag:

$$
\begin{aligned}
& D_{x}=D_{0 x}\left\{1-\frac{1}{8}\left[\frac{\alpha}{N^{2}}\left(U^{\prime 2}+2 U U^{\prime \prime}\right)+\frac{1-\alpha}{N^{2}}\left(V^{\prime 2}+4 V V^{\prime \prime}\right)\right]\right\}-\frac{1}{4} D_{0 y} \frac{1-\alpha}{N^{2}} \frac{B}{C}\left(U^{\prime} V^{\prime}+V U^{\prime \prime}\right) \\
& D_{y}=D_{0 y}\left\{1-\frac{1}{8}\left[\frac{\beta}{N^{2}}\left(V^{\prime 2}+2 V V^{\prime \prime}\right)+\frac{1-\beta}{N^{2}}\left(U^{\prime 2}+4 U U^{\prime \prime}\right)\right]\right\}-\frac{1}{4} D_{0 x} \frac{1-\beta}{N^{2}} \frac{C}{B}\left(U^{\prime} V^{\prime}+U V^{\prime \prime}\right)
\end{aligned}
$$

where $\left(D_{0 x}, D_{0 y}\right)$ are the two components of Phillips (1984) drag in a coordinate system aligned with the mountain axis. $(U, V)$ are the corresponding wind components at the surface, $\left(U^{\prime}, V^{\prime}\right)$ and $\left(U^{n}, V^{\prime \prime}\right)$ are, respectively, their first and second vertical derivatives, and $N$ is the Brunt-Väisälä frequency. $\alpha(\gamma), \beta(\gamma), C(\gamma)$ and $B(\gamma)$, are functions of the horizontal aspect ratio $\gamma$ of the mountain, defined in Teixeira and Miranda (2006). Expression (1) is equivalent to expressions (31-32) in Teixeira and Miranda (2006), although it has been modified to avoid an apparent singularity when one of the wind components goes to zero at the surface, resulting from the use of unsheared hydrostatic drag as a scaling factor in those expressions.

The previous results indicate that shear modifies the drag intensity and also its direction, when compared with unsheared flow with the same low level wind and stability. On the other hand, because the contribution of the first derivatives of velocity is squared in (1), it is easy to 
show that constant shear contributes to GWD reduction, whereas the wind profile curvature terms can contribute also to drag enhancement. The overall result depends on the vertical wind profile. The present study is, as far as we know, the first attempt to assess the relative importance of these two opposing effects with real global scale wind.

In the present study, (1) was used to compute the surface GWD, at each ECMWF grid point in the regular ERA-40 grid. To focus on the effect of shear, the computation used the available ECMWF parameter database, which includes all subgrid-scale topographic data, all atmospheric parameters needed to compute the linear unsheared drag vector $\left(D_{0 x}, D_{0 y}\right)$, and the shape functions $(\gamma, \alpha(\gamma), \beta(\gamma), B(\gamma), C(\gamma))$. Therefore, we only had to compute, for each grid point, the stability $N^{2}$, the wind velocity, and its vertical derivatives. As pointed out by Teixeira et al. (2004), the determination of the level where these quantities are computed is non-trivial issue. Following Lott and Miller (1997), the near-surface wind was computed as the average in a layer with a vertical depth equal to the standard deviation of the subgridscale orography height, $\sigma$, and a base at the same distance from the model surface, e.g. for each component, $\bar{v}=\frac{1}{\sigma} \int_{\sigma}^{2 \sigma} v d z$. The three model levels near the centre of that layer were used to estimate the wind derivatives by centred finite differences.

The nonlinear components of the ECMWF GWD scheme were not computed, as we do not have a theory to link them with shear effects. However the full ECMWF GWD from reanalysis, which includes all effects (but no shear) was retrieved for comparison. 


\section{Results}

Figure 1 shows the annual mean linear GWD stress including shear effects, for the last 10 years of ERA-40, together with a distribution of the impact of shear, given by the anomaly in the drag magnitude. Results for the full reanalysis period (not shown) are quite similar. The results highlight, as expected, the major mountain ranges, where one finds, by far, the largest stress values, and, in most cases, also the largest shear anomalies. Because GWD responds strongly to the low level wind field, the distribution of stress at any given point shows a strong annual cycle. This is clearly shown in Figure 2, presenting time series of the monthly mean linear axial GWD torque,

$$
T_{G}=\int_{\lambda=0}^{2 \pi} \int_{\phi=-\pi / 2}^{\phi=\pi / 2} \tau_{x} R^{3} \cos ^{2} \phi d \phi d \lambda
$$

where $\tau_{\mathrm{x}}$ is the zonal component of the GWD stress, $R$ is the Earth radius, $\phi$ is the latitude and $\lambda$ is the longitude. Figure 2 also shows the torque anomaly due to shear. Maximum values of linear GWD torque, of the order of $15 \mathrm{Ha}\left(1 \mathrm{Ha}=10^{18} \mathrm{~J}\right)$, are found in the Northern Hemisphere Winter, with the lowest values, near $0 \mathrm{Ha}$, in the Northern Hemisphere Summer. The impact of shear on the global torque is found to be always negative, contributing to a reduction of the mean westerly GWD torque, but its mean impact is small, at most $-1 \mathrm{Ha}$.

However, the impact seems to vary qualitatively between regions, as shown in Figure 3. In the northern sector of the Andes (NA in Figure 1 and Figure 3) although linear GWD is very large, we do not find significant impacts of shear, in the decadal mean GWD. In the Tibetan plateau (Ti), where drag is also very significant, the anomalies are distributed between negative and positive features (see Figure 1), essentially cancelling their effect at the regional scale. In the Rocky mountains (Rk) and in the southern sector of the Andes (SA) there is also a large degree of cancellation between positive and negative shear effects. That is not the case 
of Eastern Africa (Af) and especially of Antarctica (An), where shear enhances the mean GWD stress fields by a significant amount, which goes up to 50\% in the case of Antarctica.

Because both the mountains of East Africa and Antarctica experience mean easterly flow, the significant enhancement of GWD stress found there contributes to a reduction of the global westerly torque due to GWD. In the case of the southern Andes, while the relative impact of shear is rather small, it also leads, on the monthly mean, to a reduction of westerly torque but only in the peaks of its annual cycle. By contrast, the impact of shear in the Rockies leads to a slight increase in the westerly torque at the bottom of the corresponding annual cycle.

The change in global GWD torque due to shear (Figure 2) is largely a result of the contributions from East Africa and Antarctica. It consists of a small reduction of the global westerly GWD torque, due to an increase of local easterly torques in the two regions. Figure 2 also shows the global GWD torque retrieved from ERA-40, which includes parametrized non-linear effects. On a multi-annual average (not shown), the two curves are not as different as found for each year. ECMWF GWD presents large monthly oscillations not found in the linear results, either with or without shear.

Another view of the regional, and global, impact of shear in the GWD torque is shown in Figure 4, presenting distributions of shear anomalies computed from $6 \mathrm{~h}$ time series of GWD torque. Instantaneous global anomalies (outside the limits of the graph) can be up to $\pm 8 \mathrm{Ha}$, comparable with the amplitude of the annual cycle of the GWD torque, and the global anomaly is negative more than $75 \%$ of the time. The largest individual contribution to the computed anomalies comes from the East Africa mountains, contributing to reduce the westerly torque $95 \%$ of the time, with a peak $-3.5 \mathrm{Ha}$ (outside the graph). The anomalies of Antarctica are also negative $95 \%$ of the time, whereas those of the Northern Andes and Central America regions are negative $75 \%$ of the time. In the other regions, anomalies can 
frequently be of both signs, and as one would expect the largest instantaneous anomalies tend to be produced in regions with the largest mean torque values, such as Tibet or the Rocky mountains.

The latitudinal distribution of GWD torque, and of the corresponding shear anomaly, are shown in Figure 5, where decadal means were computed for the full annual cycle and for the two extreme seasons DJF and JJA. The results emphasize the well-known strong annual cycle of GWD (Oort and Peixoto, 1983), but also the fact that the shear anomaly experiences a significant annual oscillation. While the mean shear anomaly is negative (easterly), there is some positive (westerly) anomaly in the Northern Hemisphere midlatitudes, especially in the $\mathrm{NH}$ winter, when the drag is also more intense. South of $30^{\circ} \mathrm{N}$, the anomaly is mostly negative, either due to a reduction of the zonal mean westerly torque or due to the enhancement of zonal mean easterly torque. The relevance of Antarctica's anomaly is clear, considering the fact that the torque of Antarctica is quite small, due to its relatively small distance to the Earth's rotation axis.

The impact of shear on Antarctic GWD is important throughout the year, but is at its peak in the southern winter (JJA). The reason is clear in Figure 6, where the monthly mean surface flow for July 2001 is superimposed on the monthly mean flow at about $1 \mathrm{~km}$ above the surface (between $800 \mathrm{~m}$ and $1 \mathrm{~km}$ in the continental slope). At the surface, ERA-40 fields have a clear signature of the katabatic wind, a major permanent feature of the Antarctic climate. Aloft, the flow turns counter-clockwise by a large angle, implying strong wind curvature, i.e. large $\left(U^{\mathrm{n}}, V^{\mathrm{n}}\right)$. At the continental margins, where the katabatic flow accelerates and the subgrid-scale topographic variance is higher, one observes large impacts on the GWD stress field. 


\section{Discussion and conclusions}

This paper presents, as far as we know, the first attempt to compute the impact of wind profile shear and curvature on gravity wave drag, using real large scale wind fields from reanalysis. Theoretical results (Teixeira and Miranda, 2004, 2006) showed that impact of wind shear on GWD depends qualitatively on the wind profile, and may either lead to GWD reduction or enhancement. In a given location, because the flow changes in time, one may expect periods of drag enhancement and reduction to alternate. Consequently, one may expect significant cancellation of shear effects between regions, and at the same location between different time periods, when one looks at long-term averages of GWD.

However, the present results indicate that, while cancellation does occur in many places, the overall effect of shear is clearly in the sense of a reduction of the global westerly GWD torque. The impact is modest but is in the right direction, considering the imbalance currently found in the reanalysis fields (Huang et al., 1999; Egger et al., 2007).

A significant fraction of change in the global torque comes from Antarctica, where the katabatic flow guarantees a high degree of steadiness, leading to a disproportionate contribution to the impact of shear from that region. At this continental scale, shear seems to be a major contributor to the momentum balance of the Antarctic region, being responsible for an increase of about 50\% in GWD over the area. One would expect such contribution to be essential in the dynamics of the polar vortex.

Both in Antarctica and in East Africa, shear leads to the enhancement of local easterly GWD stress, indicating that the wind profile curvature effects dominate in the computation of the overall shear correction, a result that could not be easily predicted from theory. In other major mountain ranges, which are responsible for larger fractions of the total torque, shear effects tend to cancel, either due to coexistence of nearby positive and negative regions of drag 
variation or to time changes of the flow. However, the instantaneous effects of shear are likely to be much larger than those shown here for long term means.

The present study only considered the direct local effect of shear (and wind profile curvature) on surface GWD. Previous numerical (Bacmeister and Pierrehumbert, 1988; Miranda and Valente, 1997) and theoretical results (Teixeira et al., 2005, 2008) clearly indicate that surface GWD may be strongly affected by far-field effects in sheared flows, such as abrupt changes in wind shear well away from the surface, and critical levels. Those effects are not accounted for by the WKB approach used here.

It is also important to keep in mind that the wind field used in the present study came from a reanalysis database. Therefore, it was indirectly affected by the GWD scheme included in the ECMWF model at that time. A full analysis of the impact of shear on GWD could only be carried out by incorporating an alternative parametrization in the model, including the shear effect, and re-running the analysis cycle. On the other hand, while reanalyses fields are arguably the best available information on global atmospheric data, many of its details are difficult to validate against observations, namely in data-sparse regions. Bromwich et al. (2007) assessed the quality of reanalyses fields in Antarctica, including ERA-40, concluding for a good match of those fields with available observations in the latest decades (after 1979). However, they only considered synoptic scale free atmosphere circulation features, without looking into the details of near surface wind profiles required for GWD assessment.

Another difficulty in the validation of GWD estimations comes from the fact that models typically deal with 3 different subgrid-scale drag effects (surface friction, form drag and GWD), each with its own uncertainties, contributing to the global momentum balance. These different subgrid-scale drag sources respond to the low level flow in qualitatively different ways, leading to impacts in the flow at different levels. However, changes in one of the terms 
may be partially compensated in the others, making the process of parametrization a complex tuning between competing terms that can only be done in a complete model.

Finally, a sensitivity test to the present results was performed, by using an alternative method of selection of the effective wind level for GWD computation. One may argue that, considering the fact that the GWD theory is inviscid, the estimation of the parameters used to compute the surface drag should be done at the lowest level not significantly affected by boundary layer processes. Unfortunately that is not an easy definition in orographic flow. As a first approximation, instead of using the model level that is away from the surface by 1 standard deviation of the subgrid scale orography height, as used by ECMWF, we selected a constant model level, at about 500m from the surface, similar to what was done by Gregory et al. (1998). The overall mean GWD is, in that case, significantly reduced (by almost a factor of 2). However the impact of shear is quite similar in relative terms, with a reduction of westerly torque, partially due to an enhanced easterly torque in Antarctica and East Africa. This result, i.e. the weak dependence of the shear correction on the level used to compute shear and curvature, may seem surprising, considering the fact that equation (1) does not give a clear indication of the sign of the shear correction. However, qualitative arguments, briefly described in the Appendix, indicate that in the real world, where the wind field cannot have an unbounded shear layer, one should expect, on average, a dominant positive impact on the GWD due to the wind profile curvature.

Acknowledgements. The present study was supported by the Calouste Gulbenkian Foundation, and by Fundação para a Ciência e Tecnologia (FCT) under Project BOSS, Contract POCI/CTE-ATM/58932/2004, co-financed by the European Union under program FEDER. François Lott and Pedro Viterbo helped in the interpretation of ERA-40 drag fields. 
Appendix

Why do curvature effects dominate in the computation of the shear correction to GWD drag? The answer is not easy in the general case. However, some hints may be obtained for the special case of GWD produced by a ridge. In that case, equation (1) simplifies to:

$D_{x}=D_{x 0}\left\{1-\frac{1}{8} \frac{U r^{2}}{N^{2}}-\frac{1}{4} \frac{U U^{\prime \prime}}{N^{2}}\right\}$

If one integrates the shear correction in an arbitrary atmospheric layer, one obtains after some algebra:

$\int_{z_{1}}^{z_{2}} \frac{D_{x}}{D_{x 0}} d z=\int_{z_{1}}^{z_{2}}\left\{1-\frac{1}{8} \frac{U^{\prime 2}}{N^{2}}-\frac{1}{4} \frac{U U^{\prime \prime}}{N^{2}}\right\} d z=\left(z_{2}-z_{1}\right)+\frac{1}{8} \int_{z_{1}}^{Z_{2}} \frac{1}{R i} d z+\frac{1}{4}\left[\left(\frac{U U \prime}{N^{2}}\right)_{z_{1}}-\left(\frac{U U \prime}{N^{2}}\right)_{z_{2}}\right]$

From (A2) one may compute the vertical mean correction in that layer:

$\overline{\left(\frac{D_{x}}{D_{x 0}}\right)}=\frac{1}{z_{2}-z_{1}} \int_{z_{1}}^{z_{2}} \frac{D_{x}}{D_{x 0}} d z=1+\frac{1}{8} \overline{R l^{-1}}+\frac{1}{4} \frac{1}{z_{2}-z_{1}}\left[\left(\frac{U U I}{N^{2}}\right)_{z_{1}}-\left(\frac{U U I}{N^{2}}\right)_{z_{2}}\right]$

Because $U U^{I} / N^{2}$ may be positive or negative (and must remain bounded), the last term will

not give a definite contribution to the drag correction (especially for large $z_{2}-z_{1}$ ), implying that the correction will in general be positive. The consequence is that, if the level where the wind velocity and its derivatives are evaluated is chosen randomly over a range of heights between $z_{1}$ and $z_{2}$, the drag is more likely to be enhanced than to be reduced. This means that the tendency for drag enhancement is, to a certain extent, independent of the chosen level. A qualitatively similar, but yet more striking, result might be derived for 3D orography, e.g. in 
the case of a circular mountain, where wind turning effects further enhance the drag (Teixeira et al. 2004).

It should be stressed that the previous argument does not take into account the possibility of a critical level below $z_{2}$, as in that case the drag may not be affected by the wind profile above that level due to wave attenuation effects (cf. Teixeira et al. 2008). 
References

Bacmeister JT, Pierrehumbert RT. 1988. On high-drag states of nonlinear stratified flow over an obstacle. J. Atmos. Sci., 45, 63-80. DOI: 10.1175/1520-0469.

Doyle, JD, Jiang QJ. 2006. Observations and numerical simulations of mountain waves in the presence of directional wind shear. Q. J. R. Meteorol. Soc., 132, 1877-1905, DOI: 10.1256/qj.05.140.

Egger J, Weickmann K, Hoinka KP. 2007. Angular momentum in the global atmospheric circulation, Rev. Geophys., 45, RG4007. DOI:10.1029/2006RG000213, DOI: 10.1002/qj.49712454606.

Gregory D, Shutts GJ, Mitchell JR. 1998. A new gravity-wave-drag scheme incorporating anisotropic orography and low-level wave breaking: impact upon the climate of the UK Meteorological Office Unified Model. Q. J. R. Meteorol. Soc., 124, 463-493. DOI: 10.1002/qj.49712454606. DOI: 10.1256/qj.04.160.

Huang HP, Sardeshmukh P, Weickmann K. 1999. The balance of global angular momentum in a long-term atmospheric data set. J. Geophys. Res., 104, 2031-2040.Kim, Y. -J. 2007. Balance of drag between the middle and lower atmospheres in a global atmospheric forecast model, J. Geophys. Res., 112, D13104, doi:10.1029/2007JD008647.

Kim, Y. -J., Arakawa A. 1995. Improvement of orographic gravity wave parameterization using a mesoscale gravity-wave model. J. Atmos. Sci., 52, 1875- 1902. 
Kim YJ, Doyle JD. 2005. Extension of an orographic-drag parametrization scheme to incorporate orographic anisotropy and flow blocking. Q. J. R. Meteorol. Soc., 131, 18931921, DOI:10.1256/qj.04.160.

Lindzen RS. 1981. Turbulence and stress owing to gravity wave and tidal breakdown. $J$. Geophys. Res., 86, 9707-9714.

Lott F, Miller MJ. 1997. A new subgrid-scale orographic drag parameterization: Its formulation and testing. Q. J. R. Meteorol. Soc., 123, 101-127. DOI: 10.1002/qj.49712353704.

McFarlane NA. 1987. The effect of orographically excited gravity-wave drag on the general circulation of the lower stratosphere and troposphere. J. Atmos. Sci., 44, 1775-1800.

Miranda PMA, IN James. 1992. Nonlinear three-dimensional effects on gravity waves: Splitting flow and breaking waves. Q. J. R. Meteorol. Soc., 118, 1057-1081. DOI: 10.1002/qj.49711850803 .

Miranda PMA, Valente MA. 1997. On Critical Level Resonance in Three-dimensional Flow Past Isolated Mountains, J. Atmos. Sci., 54, 1574-1588.

Palmer TN, Shutts G, Swinbank R. 1986. Alleviation of a systematic westerly bias in general circulation and numerical weather prediction models through an orographic gravity wave parameterization. Q. J. R. Meteorol. Soc., 112, 1001-1039. DOI: 10.1002/qj.49711247406.

Oort A, Peixoto J. 1983. Global angular momentum and energy balance requirements from observations. Adv. Geophys., 25, 355-490.

Phillips DS. 1984. Analytical surface pressure and drag for linear hydrostatic flow over threedimensional elliptical mountains. J. Atmos. Sci., 41, 1073-1084. 
Sawyer JS. 1959. The introduction of the effects of topography into methods of numerical forecasting. Q. J. R. Meteorol. Soc., 85, 31-43.

Shutts GJ, Gadian A. 1999. Numerical simulations of orographic gravity waves in flows which back with height. Q. J. R. Meteorol. Soc., 125, 2743-2765. DOI: 10.1002/qj.49712555920.

Smith RB, Doyle JD, Jiang Q, Smith SA. 2007. Alpine gravity waves: Lessons from MAP regarding mountain wave generation and breaking. Q. J. R. Meteorol. Soc., 133, 917-936. DOI: 10.1002/qj.103.

Stein J. 1992. Investigation of the regime diagram of hydrostatic flow over a mountain with a primitive equation model. Part I: Two dimensional flows. Mon. Weather Rev., 120, 29622976.

Teixeira MAC, Miranda PMA. 2004. The effect of wind shear and curvature on the gravity wave drag produced by a ridge. J. Atmos. Sci., 61, 2638-2643.

Teixeira MAC, Miranda PMA. 2006. A linear model of gravity wave drag for hydrostatic sheared flow over elliptical mountains. Q. J. R. Meteorol. Soc., 132, 2439-2458.DOI: 10.1256/qj.05.220.

Teixeira MAC, Miranda PMA, Argain JL. 2008. Mountain waves in two-layer sheared flows: critical level effects, wave reflection and drag enhancement. J. Atmos. Sci., 65, 1912-1926.

Teixeira MAC, Miranda PMA, Valente MA. 2004. An analytical model of mountain wave drag for wind profiles with shear and curvature. J. Atmos. Sci., 61, 1040-1054.

Uppala S, and coauthors. 2005. The ERA-40 re-analysis. Q. J. R. Meteorol. Soc., 131, 29613012. DOI: 10.1256/qj.04.176. 
Wallace JM, Tibaldi S, Simmons AJ. 1983. Reduction of systematic errors in the ECMWF model through the introduction of an envelope orography. Q. J. R. Meteorol. Soc., 109, 683718. 
Figure 1 Annual mean linear GWD stress (1992-2001): surface stress with shear (vectors), anomaly of the modulus of stress due to shear (shaded). Gray rectangles indicate areas used to evaluate mountain range torque.

Figure 2 Monthly mean GWD torque (Hadley): (top) global torque anomaly due to shear, (bottom) computed global GWD torque with shear (blue curve) and ERA-40 GWD torque including also non-linear effects (red curve).

Figure 3 Monthly mean linear GWD torque (Hadley) with shear (red) and without (black) in large mountainous regions: Gr (Greenland), Eu (Europe), Ti (Tibet), Rk (Rockies), ME (Middle East), Af (East Africa), CA (Central America), NA (Northern Andes), SA (Southern Andes), R (Remaining area), cf. Figure 1. Note that the scales vary.

Figure 4 - Distribution of 6-hourly torque anomaly due to shear: small squares are the data mean, rectangles delimit the $25 \%$ and $75 \%$ region, horizontal lines inside the rectangles locate the median, vertical lines extend to the $5 \%$ and $95 \%$, crosses at $1 \%$ and $99 \%$.

Figure 5 - Seasonal-mean GWD axial torque (left curves), and torque anomaly due to shear (right). Solid lines: linear torque and anomaly computed in this study: annual mean (black), DJF (blue), JJA (red). Dotted lines: surface GWD torque (including parametrized nonlinear effects) from ERA-40.

Figure 6 - Mean July 2001 wind field in Antarctica: lowest model level (level 60, black arrows), level 50 (at about $1 \mathrm{~km}$ above the surface, magenta arrows). Colour shades: modulus of GWD stress anomalies due to shear, in $\mathrm{Pa}$. 


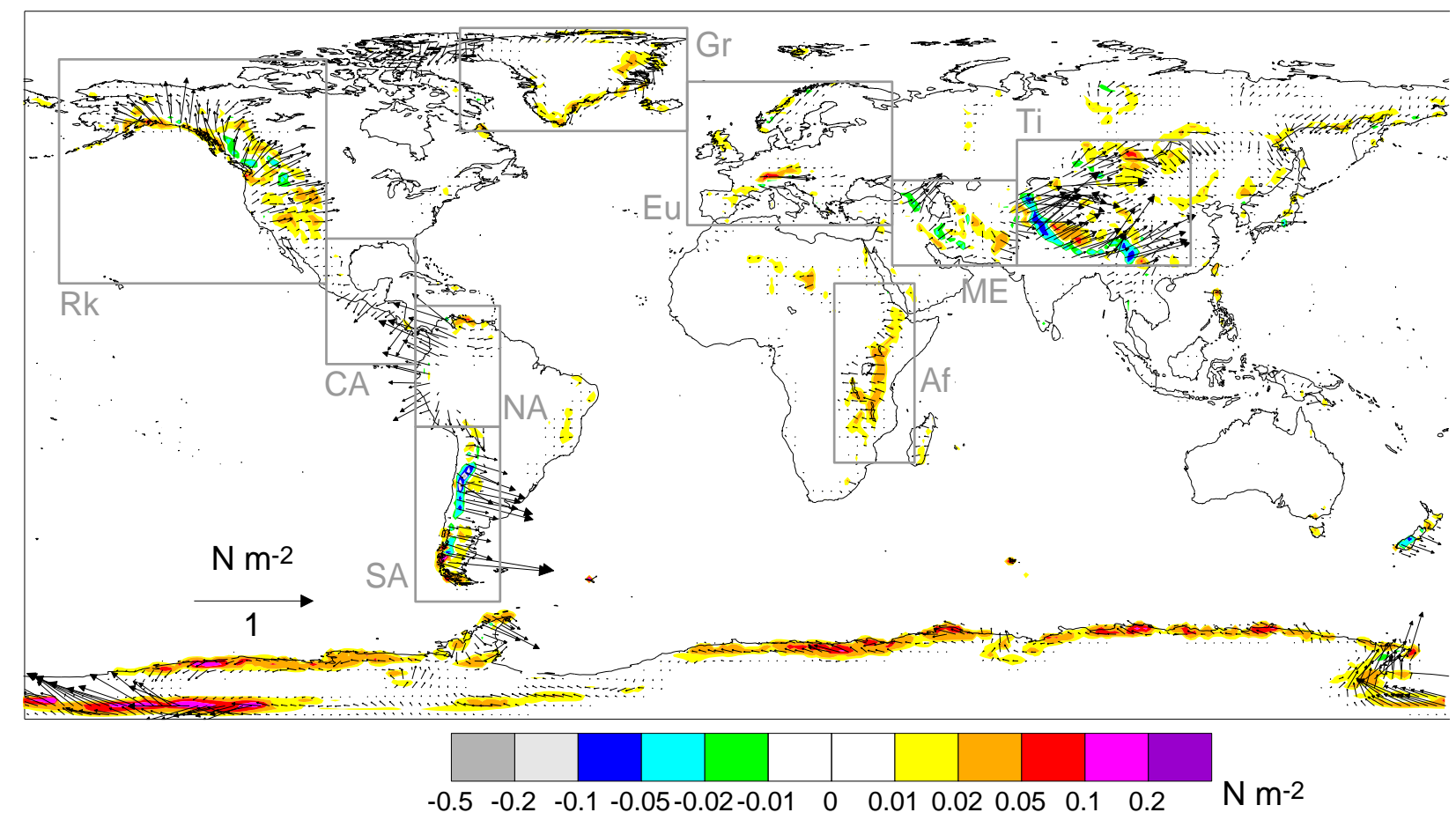

Figure 1 Annual mean linear GWD stress (1992-2001): surface stress with shear (vectors), anomaly of the modulus of stress due to shear (shaded). Gray rectangles indicate areas used to evaluate mountain range torque. Computed from ERA-40 data at $1.125^{\circ} \times 1.125^{\circ}$. 


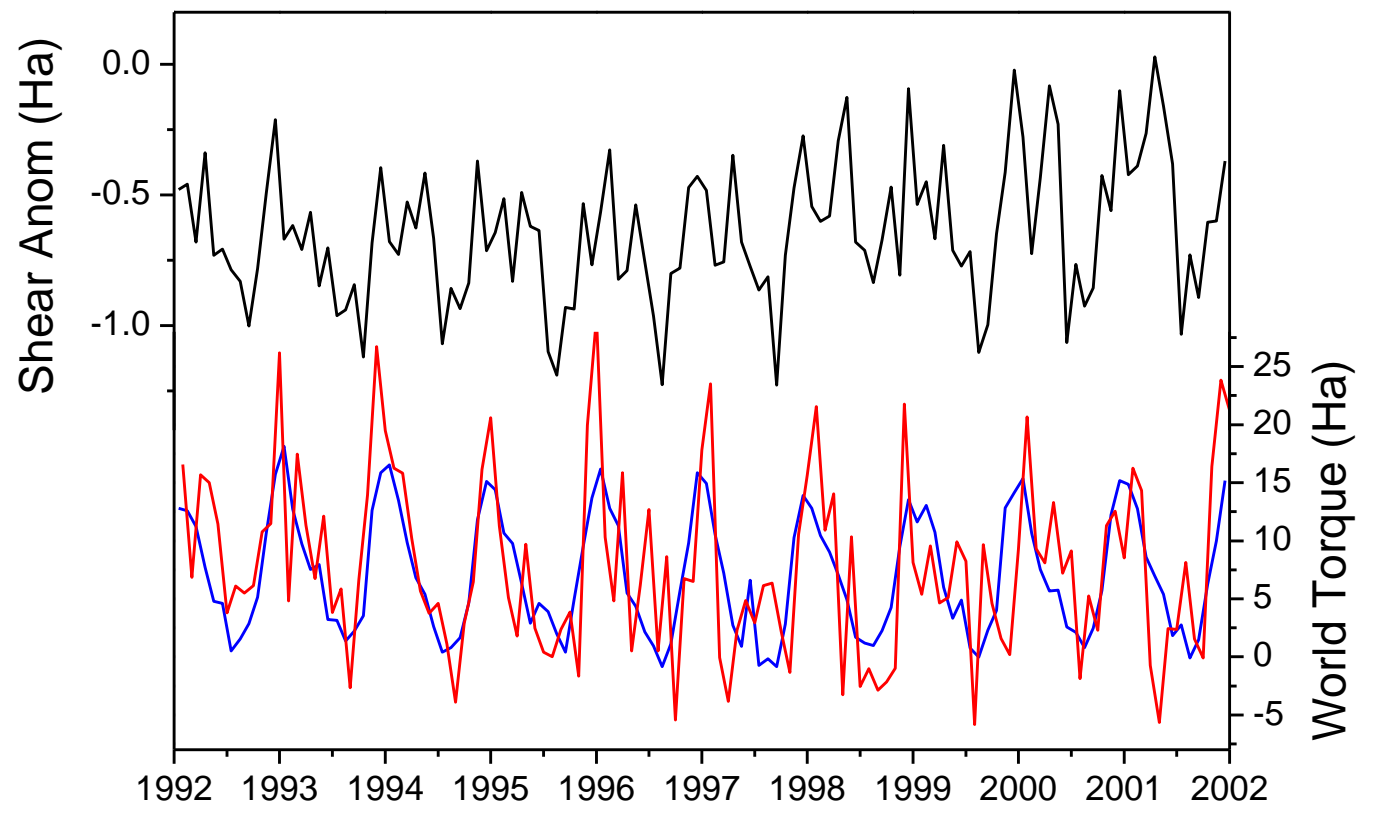

Figure 2 Monthly mean GWD torque (Hadley): (top) global torque anomaly due to shear, (bottom) computed global GWD torque with shear (blue curve) and ERA-40 GWD torque including also non-linear effects (red curve). 


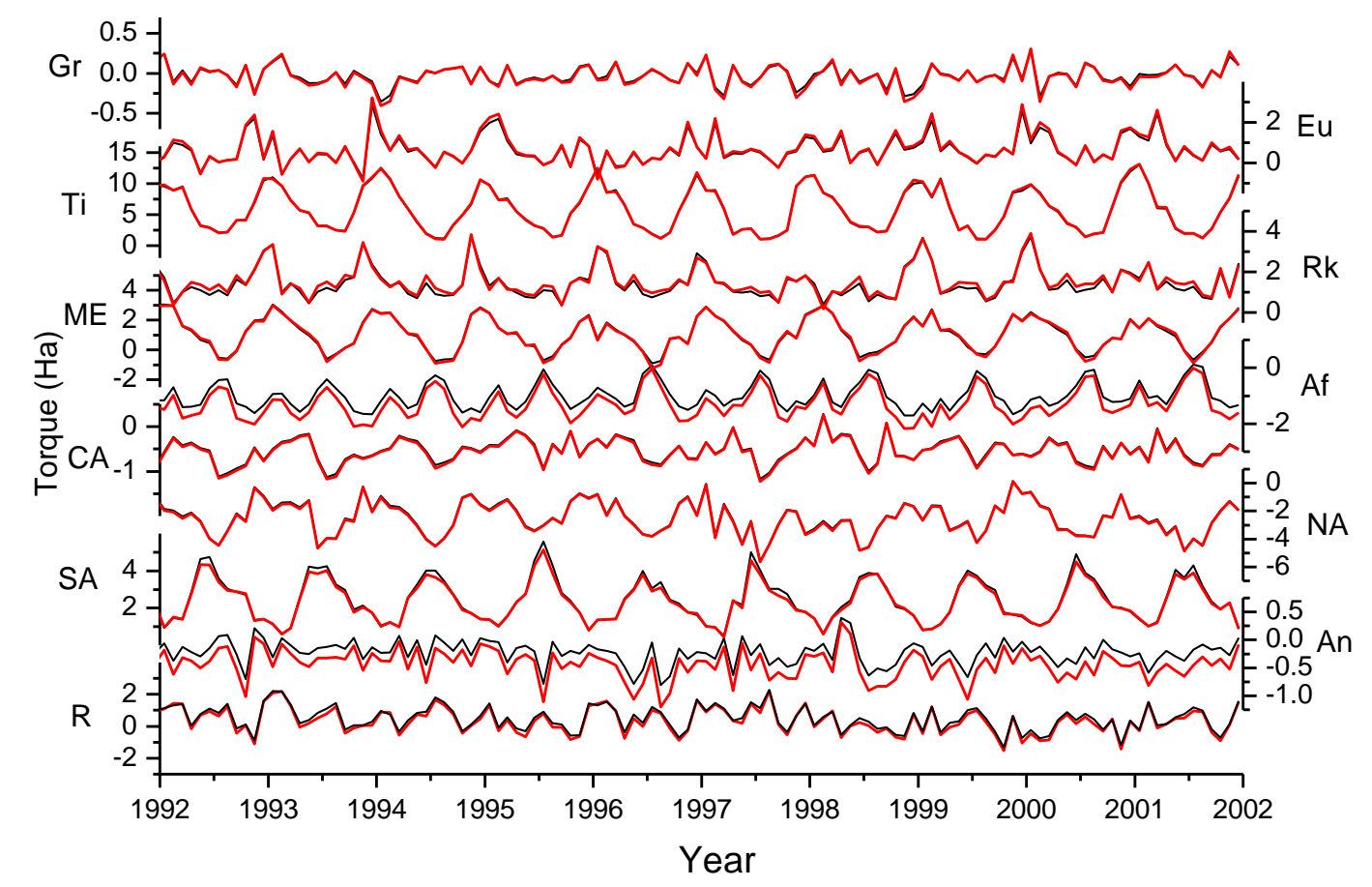

Figure 3 Monthly mean linear GWD torque (Hadley) with shear (red) and without (black) in large mountainous regions: Gr (Greenland), Eu (Europe), Ti (Tibet), Rk (Rockies), ME (Middle East), Af (East Africa), CA (Central America), NA (Northern Andes), SA (Southern Andes), R (Remaining area), cf. Figure 1. Note that the scales vary. 


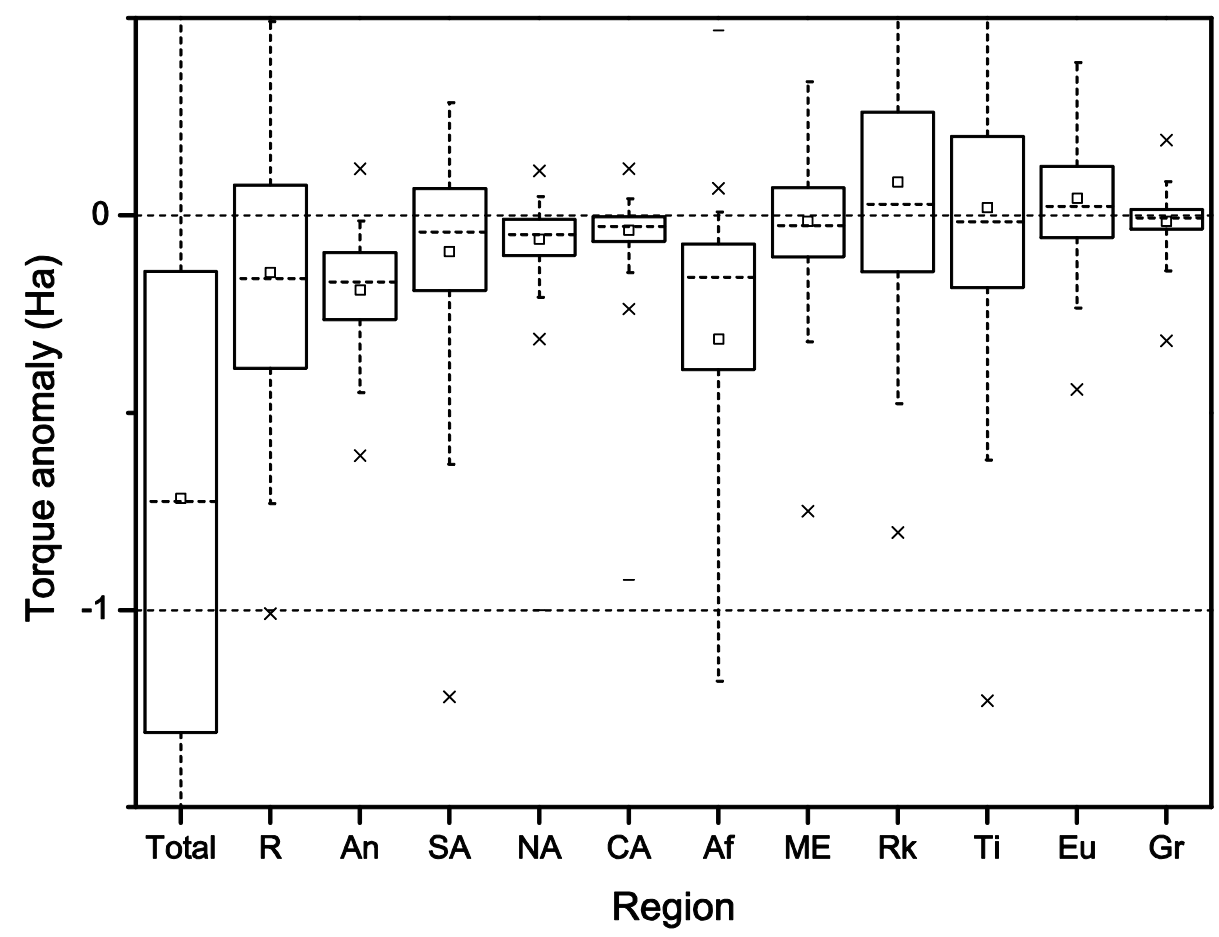

Figure 4 - Distribution of 6-hourly torque anomaly due to shear: small squares are the data mean, rectangles delimit the $25 \%$ and $75 \%$ region, horizontal lines inside the rectangles locate the median, vertical lines extend to the $5 \%$ and $95 \%$, crosses at $1 \%$ and $99 \%$. 


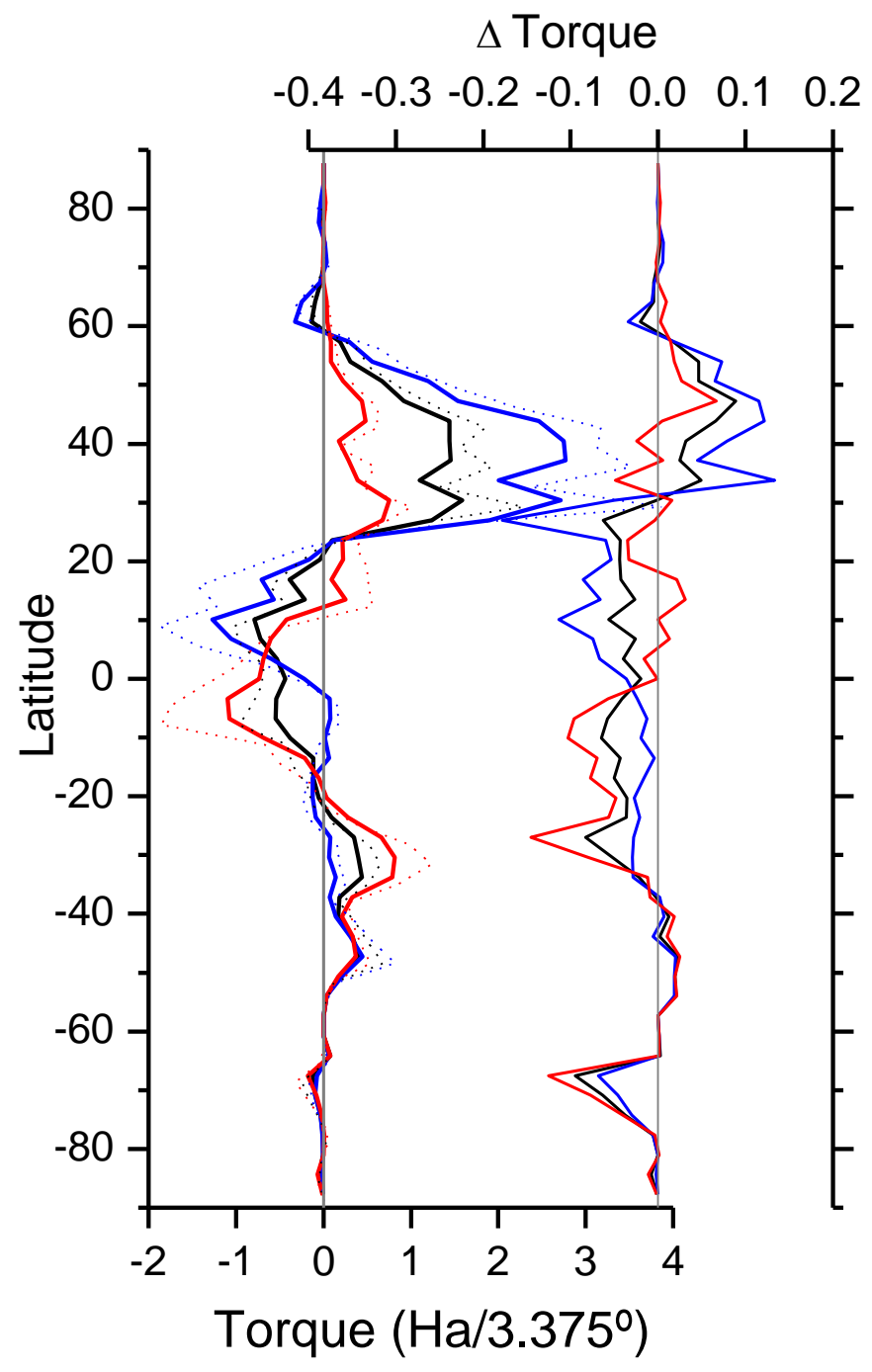

Figure 5 - Seasonal-mean GWD axial torque (left curves), and torque anomaly due to shear (right). Solid lines: linear torque and anomaly computed in this study: annual mean (black), DJF (blue), JJA (red). Dotted lines: surface GWD torque (including parametrized nonlinear effects) from ERA-40. 


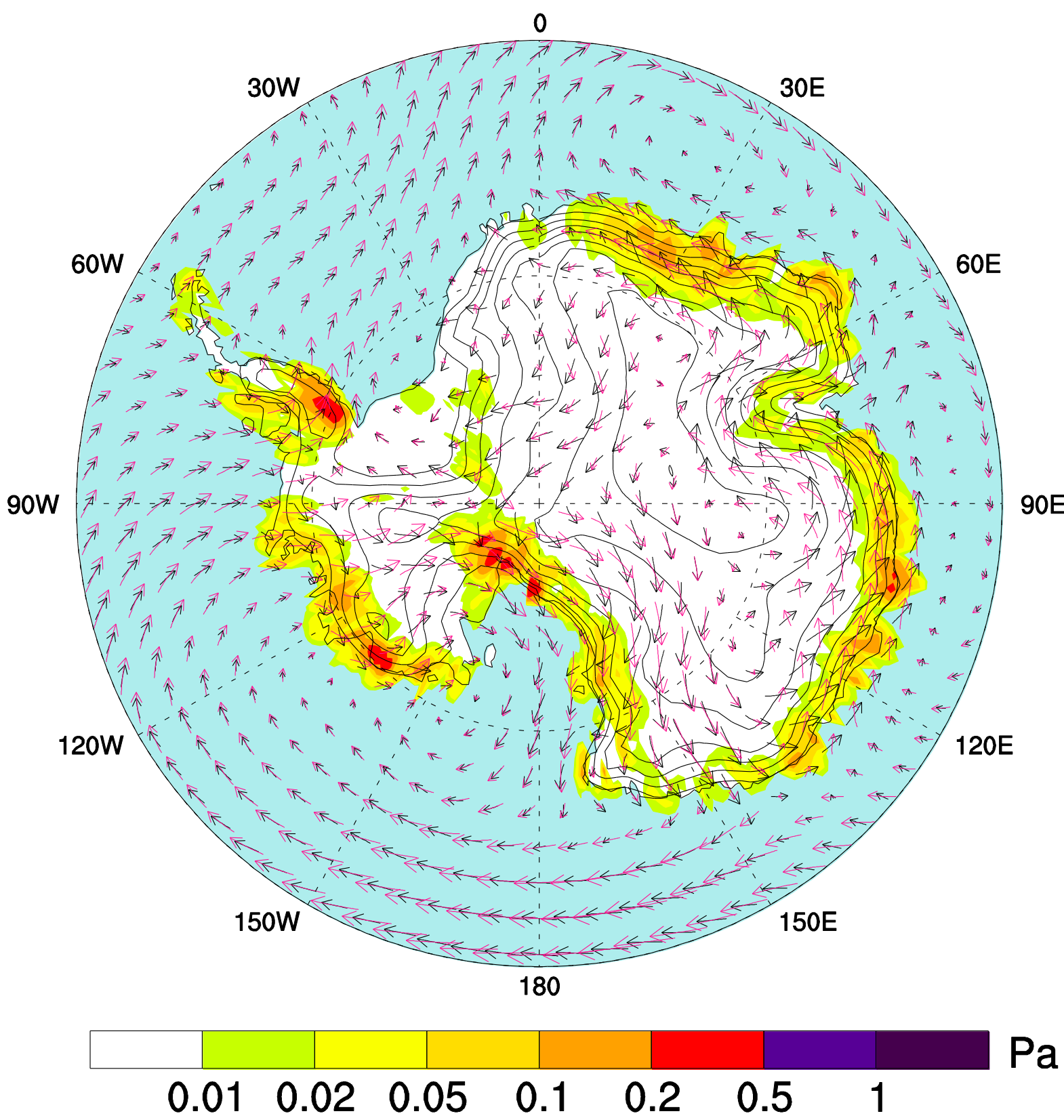

Figure 6 - Mean July 2001 wind field in Antarctica: lowest model level (level 60, black arrows), level 50 (at about $1 \mathrm{~km}$ above the surface, magenta arrows). Colour shades: modulus of GWD stress anomalies due to shear, in Pa. Mean topography contour levels (black lines) every $500 \mathrm{~m}$. 